\title{
BOUNDARY VALUE PROBLEMS IN AERODYNAMICS OF LIFTING SURFACES IN NON-UNIFORM MOTION
}

\author{
ERIC REISSNER
}

1. Introduction. In the present paper we propose to discuss certain aspects of the theory of lifting surfaces in non-uniform motion. Briefly, lifting surface theory is concerned with the motion of an impenetrable, deformable surface through an incompressible or compressible non-viscous fluid. In general the impenetrable surface is intended to represent approximately an airplane wing, a tail surface, or a propeller. The adjective lifting indicates the nature of the interaction desired between the impenetrable surface and the surrounding fluid.

The mathematical nature of the problems arising in this theory is that of boundary value problems of partial differential equations. Our principal object here is formulation of these boundary value problems and presentation of some of the methods, exact or approximate, which have been used in the solution of some of these problems. As may be seen from the list at the end of this paper the amount of work done in this field is considerable and the following account is restricted to those aspects of the theory which have been of particular interest to the writer.

Lifting surface theory as developed may be designated as a perturbation theory in the following sense. Because of the assumption of no viscosity there are evidently types of motion of an impenetrable surface which proceed without disturbing the surrounding fluid at all. One now asks for such motions which proceed nearly without producing any disturbances and one uses the assumption of small disturbances to simplify the differential equations and boundary conditions of the theory. In general this simplification leads to a linearized theory and it is this linearized theory which will here be discussed. The main reason for the considerable literature on the subject is the fact that the range of applicability of the linearized theory has been found adequate for many problems arising in engineering, and in particular in aeronautical engineering.

Evidently one may, if one wishes, consider separately problems of uniform and non-uniform motion in lifting surface theory. Histori-

An address delivered before the New York Meeting of the Society on February 28, 1948, by invitation of the Committee to Select Hour Speakers for Eastern Sectional Meetings; received by the editors November 12, 1948. 
cally, uniform-motion theory, as initiated by Prandtl, precedes nonuniform motion theory by about ten years. Solution of problems of non-uniform motion theory has turned out to be of considerably greater mathematical complexity than solution of uniform-motion problems.

In what follows we shall formulate the problems of non-uniform motion in somewhat greater generality than has heretofore been done. After this we shall discuss in some detail various aspects of the theory of nearly plane lifting surfaces in incompressible flow, and in particular the step from two-dimensional to three-dimensional theory.

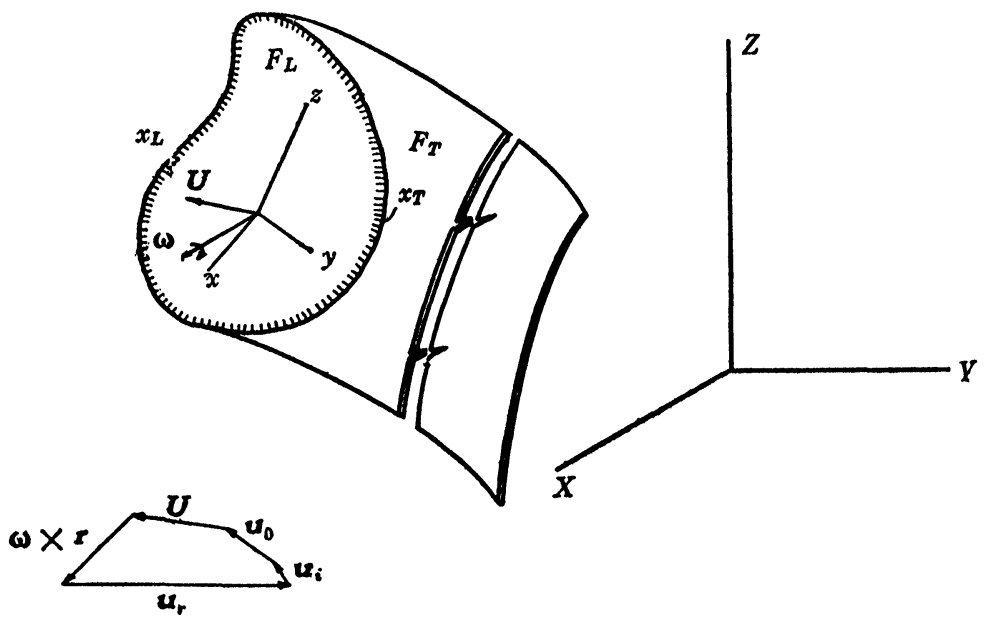

Fig. 1

2. The general problem. The actual problem of linearized lifting surface theory will be considered as an approximation to the following nonlinear problem. An impenetrable, deformable surface of given extent moves in a prescribed manner through a compressible perfect fluid. From part of the edge of the impenetrable surface emanates a surface of velocity discontinuity in such a manner that the fluid velocity remains finite along this part of the edge, ${ }^{1}$ henceforth called the trailing edge. Along the remainder of the edge, called leading

${ }_{1}^{1}$ This condition of finite trailing edge velocity, first introduced in two-dimensional airfoil theory by Kutta and Joukovsky in order to obtain a definite lifting action, was subsequently found to represent rather well the effect of viscosity of actual fluids in a perfect-fluid theory of airfoils. The fact that in a three-dimensional theory enforcement of this condition necessitated introduction of a trailing surface of discontinuity was first observed by Prandtl. 
edge, the fluid velocity will on account of the sharpness of the edge in general assume infinite values for an incompressible fluid. For a compressible fluid the assumption of a sharp leading edge will in general make impossible a continuous solution in the region exterior to the surfaces of discontinuity. We need not for the present purposes consider this difficulty as it disappears in the linearized form of the problem.

Let $X, Y, Z$ be the axes of a fixed frame of reference and let $x, y, z$ be the axes of a frame of reference moving with the impenetrable surface (Fig. 1). Let $\boldsymbol{U}(t)$ be the velocity of the origin of the moving system with reference to the fixed system and let $\omega(t)$ be the angular velocity of the moving system with reference to the fixed system. Let $u_{r}$ be the velocity of a fluid particle relative to the moving system and let $\boldsymbol{u}$ be the velocity of the same particle relative to the fixed system. The velocity vector $\boldsymbol{u}$ may be written in the form $\boldsymbol{u}_{0}+\boldsymbol{u}_{\boldsymbol{i}}$, where $u_{0}$ exists without being caused by the presence of the impenetrable surface and where $\boldsymbol{u}_{i}$ is induced by the motion of the impenetrable surface. Correspondingly we have a pressure $p=p_{0}+p_{i}$ and a density $\rho=\rho_{0}+\rho_{i}$.

We then have the following kinematical relations involving velocity $\boldsymbol{u}$ and acceleration $\boldsymbol{a}$

$$
\begin{aligned}
& u=u_{r}+U+\omega \times r, \\
& a=(\partial u / \partial t)+u_{r} \cdot \nabla u+\omega \times u .
\end{aligned}
$$

The differential equations of the problem are of the following form

$$
\begin{gathered}
\rho a+\nabla p=0, \\
(\partial \rho / \partial t)+\nabla \cdot\left(\rho u_{r}\right)=0, \\
p=f(\rho) .
\end{gathered}
$$

Equations (3) to (5) are to be solved in the space exterior to two surfaces $F_{L}=0$ and $F_{T}=0$, where $F_{L}$ represents the given surface of pressure and velocity discontinuity and where $F_{T}$ represents a surface of velocity discontinuity, determination of which is part of the problem. On $F_{L}$ we have the condition of no relative normal flow. On $F_{T}$ we have the two conditions that the normal velocities of points of the surface are given by the corresponding velocities of the surrounding fluid and that the pressure is continuous across this surface. Thus

$$
\begin{gathered}
F_{L}(x, y, z, t)=0 ; \quad \partial F_{L} / \partial t+u_{r} \cdot \nabla F_{L}=0, \\
F_{T}(x, y, z, t)=0 ; \quad \partial F_{T} / \partial t+u_{r \pm} \cdot \nabla F_{T}=0, \quad p_{+}=p_{-},
\end{gathered}
$$

where the subscripts + and - distinguish the two sides of $F_{T}$. 
The surfaces $F_{L}$ and $F_{T}$ are connected along a line $C_{T}$ which is part of the edge of $F_{L}=0$ and which is sufficiently described for the present purposes by the designation "trailing" edge. Along $C_{T}$ we have the additional condition that $u$ remains finite.

In addition to the boundary condition (6) and (7) and the trailing edge conditions there are needed conditions at infinity. The form of these conditions will evidently depend on the form of the motion and on whether the fluid is compressible or incompressible.

For incompressible flow these conditions are roughly vanishing of all disturbances at an infinite distance from lifting surface and trailing surface. For compressible flow no such general statement can be made. In some cases all that is required is to superimpose on the conditions for imcompressible flow a condition stating that radiation energy is not created or reflected at infinity. In other cases the disturbances caused by the motion of the lifting surface cannot be required to vanish at infinity. General determination of these conditions is outside the scope of this report.

When the velocity distribution $u_{0}$ which exists without the presence of the surface $F_{L}$ is such that $\nabla \times u_{0}=0$, equations (3) to (5) may be reduced to one scalar equation by means of the introduction of a velocity potential $\phi$ which, for incompressible flow, satisfies the Laplace equation but which for compressible flow is of a more general type.

We postpone here introduction of the velocity potential and first linearize the problem.

3. The linearized form of the general problem. Basic assumption for a linearized theory is that the lifting surface moves through the fluid nearly without disturbing the fluid such that powers and products of the quantities $\boldsymbol{u}_{i}, \boldsymbol{p}_{i}$ and $\rho_{i}$ and their derivatives may be neglected.

The equation of motion (3) becomes then

$$
\begin{aligned}
\partial u_{i} / \partial t-(U+\omega \times r) \cdot \nabla u_{i}+\omega \times u_{i}+u_{i} \cdot \nabla u_{0}+u_{0} \cdot \nabla u_{i} \\
=-\frac{\nabla p_{i}}{\rho_{0}}+\frac{\nabla p_{0}}{\rho_{0}} \frac{\rho_{i}}{\rho_{0}} .
\end{aligned}
$$

The equation of continuity (4) becomes

$$
\left(\partial \rho_{i} / \partial t\right)+\nabla\left(\rho_{0} u_{i}\right)+\nabla\left[\left(u_{0}-U-\omega \times r\right) \rho_{i}\right]=0
$$

and the equation of change of state becomes

$$
p_{i}=f^{\prime}\left(\rho_{0}\right) \rho_{i} .
$$


We shall in some of what follows write as abbreviations

$$
f^{\prime}\left(\rho_{0}\right)=a_{0}^{2}, \quad U+\omega \times r=V .
$$

The quantity $a_{0}$ is the velocity of sound at a point of the undisturbed medium.

Turning now to the boundary conditions (6) and (7) we begin by establishing the condition for the motion of a surface $F_{L P}=0$ without any disturbance of the surrounding fluid. Equation (6) indicates that this condition is as follows

$$
\partial F_{L P} / \partial t+\left(u_{0}-V\right) \cdot \nabla F_{L P}=0 .
$$

We have chosen the subscript $P$ to indicate this surface because we wish to refer to it henceforth as the projection of the lifting surface. As the actual lifting surface must deviate only slightly from this projection in order to move nearly without causing disturbances we may write

$$
F_{L}=F_{L P}+f_{L}=0
$$

where $f_{L}$ is small in the same sense as $u_{i}, \rho_{i}$, and $p_{i}$ are small. Then, considering (12), and except for quantities small of higher order, the boundary condition at the lifting surface becomes

$$
F_{L P}=0 ; \quad \partial f_{L} / \partial t+\left(u_{0}-V\right) \cdot \nabla f_{L}+u_{i} \cdot \nabla F_{L P}=0 .
$$

Note that in satisfying the boundary condition at the projection of the lifting surface rather than at the lifting surface itself we again depend on the perturbation properties of the solution to be obtained.

The next step is the determination of the form of the trailing surface of discontinuity $F_{T}$. As the equation of this surface is one of the unknowns of the problem we must, in order to have a linear problem, omit the term $u_{i}$ in (7) and the shape of $F_{T}$ is then such that the equation

$$
\partial F_{T} / \partial t+\left(u_{0}-V\right) \cdot \nabla F_{T}=0
$$

is satisfied. In addition to this we have the condition that at the trailing edge $C_{T}$ the surface $F_{T}$ is connected to the surface $F_{L P}$. The meaning of (15) is that within the linearized theory the shape of the trailing surface of discontinuity is independent of the velocity distribution $\boldsymbol{u}_{i}$ induced by the motion of the lifting surface.

Having the equation of $F_{T}$ we then obtain from (17) the two conditions of continuous normal velocity and pressure across the surface in the form

$$
F_{T}=0 ; \quad\left(u_{i_{+}}-u_{i-}\right) \cdot \nabla F_{T}=0, p_{+}-p_{-}=0 .
$$


To the formulation of the problem as contained in equations (8) to (16) we must add the condition of finite $u_{i}$ along $C_{T}$ and appropriate conditions at infinity.

We remark that previous formulations of this problem of nonsteady motion in their most general form are based on the assumptions $u_{0}=0, \omega=0, U=U i$. Under this assumption, and under the additional assumptions that $d U / d t=0$ and $\partial F_{L P} / \partial t=0$, Kuessner $[44]^{2}$ has obtained an integral equation for the pressure distribution at the lifting surface.

4. Velocity potential formulation of the linearized problem. In what follows we shall assume that the fluid is at rest except for the motion induced by the lifting surface, that is, we put

$$
u_{0}=0, \quad \nabla p_{0}=0 .
$$

With these assumptions we have the existence of a velocity potential $\phi$ in terms of which

$$
u_{i}=\nabla \phi
$$

Combination of (18), (17), and (8) gives for the pressure $p_{i}$ the following expression

$$
p_{i} / \rho_{0}=(U+\omega \times \boldsymbol{r}) \cdot \nabla \phi-\partial \phi / \partial t .
$$

Combination of (19), (11), (10), and (9) gives the following differential equation for $\phi$,

$$
\nabla^{2} \phi-\left(1 / a_{0}^{2}\right)[\partial / \partial t-(U+\omega \times \boldsymbol{r}) \cdot \nabla]^{2} \phi=0 .
$$

The boundary condition (14) becomes

$$
F_{L P}=0 ; \quad \frac{\partial \phi}{\partial n}\left|\nabla F_{L P}\right|=(\boldsymbol{U}+\omega \times \boldsymbol{r}) \cdot \nabla f_{L}-\frac{\partial f_{L}}{\partial t} .
$$

The transition conditions (16) become

$$
F_{T}=0\left\{\begin{array}{l}
\left(\frac{\partial \phi}{\partial n}\right)_{+}=\left(\frac{\partial \phi}{\partial n}\right)_{-} \\
{\left[\frac{\partial \phi}{\partial t}-(U+\omega \times \boldsymbol{r}) \cdot \nabla \phi\right]_{+}} \\
=\left[\frac{\partial \phi}{\partial t}-(U+\omega \times \boldsymbol{r}) \cdot \nabla \phi\right]_{-}
\end{array}\right.
$$

${ }^{2}$ Numbers in brackets refer to the references cited at the end of the paper. 
and the trailing edge condition is that along $C_{T}$ we have $\nabla \phi$ finite.

The problem from here on is the solution of the mixed boundary value problem (20) to (22), with appropriate conditions at infinity. The object of such solutions is primarily the determination of the pressures $p_{i}$ on both sides of the lifting surface.

Solutions obtained so far are all for nearly plane lifting surfaces such that $F_{L P} \equiv z=0$. Predominant among these are solutions for the two-dimensional problem, which may be characterised by the requirement that $\partial \phi / \partial y \equiv 0$.

For two-dimensional incompressible flow essential contributions are due to $H$. Wagner [78], W. Birnbaum [3], H. Glauert [22], T. Theodorsen [73], H. G. Kuessner [42], P. Cicala [7], and G. Ellenberger [14].

For two-dimensional subsonic compressible flow which is less completely solved than the problem of incompressible flow, one must mention the work of C. Possio [53], R. Timman [75], D. Haskind [24], and that given in [60]. Various approximate methods for the solution of Possio's integral equation of the problem are described in [39].

The corresponding problem in the supersonic range has been dealt with by Possio [52], S. von Borbely [6], H. A. John and G. Temple [72], I. E. Garrick and I. Rubinov [19], and I. A. Panichkin [51].

The perturbation theory of non-steady two-dimensional transonic flow has recently been considered by C. C. Lin, H. S. Tsien and the writer [47].

In the three-dinensional theory one has the solution of Schade and Krienes $[41,62]$ for the lifting surface of circular plan-form in incompressible flow and, also for incompressible flow, a number of developments for an approximate analysis of the three-dimensionality of the flow for surfaces whose span is appreciably greater than their chord. We shall in what follows describe a particular approach to this problem based on earlier publications on this subject $[\mathbf{5 6}, \mathbf{5 7}]$. A discussion of various other methods of analysis for this problem by Cicala $[8,9]$, W. R. Sears [69], R. T. Jones [32, 33], and Kuessner [44] can be found in [56].

Finally, we mention work by Garrick and Rubinov [20] and by E. A. Krasilschikova [40] on three-dimensional supersonic flow and a forthcoming publication on three-dimensional subsonic flow [59]. In both problems it appears that further work is required before all difficulties inherent in the problem are overcome.

5. Motion of nearly plane lifting surface in incompressible flow. Further discussion will be carried out for this subclass of the general 
problem. Our object is to indicate the particular nature of the boundary value problem in question and to outline one of the possible methods of solution.

We assume that the projection of the lifting surface lies in the $x, y$-plane and that the motion of the lifting surface is in the direction of negative $x$. We further assume incompressible flow. We have then

$$
U=-U i, \quad \omega=0, \quad a_{0}=\infty
$$

and, in accordance with (12) and (15),

$$
F_{L P}=z=0, \quad F_{T}=z=0 .
$$

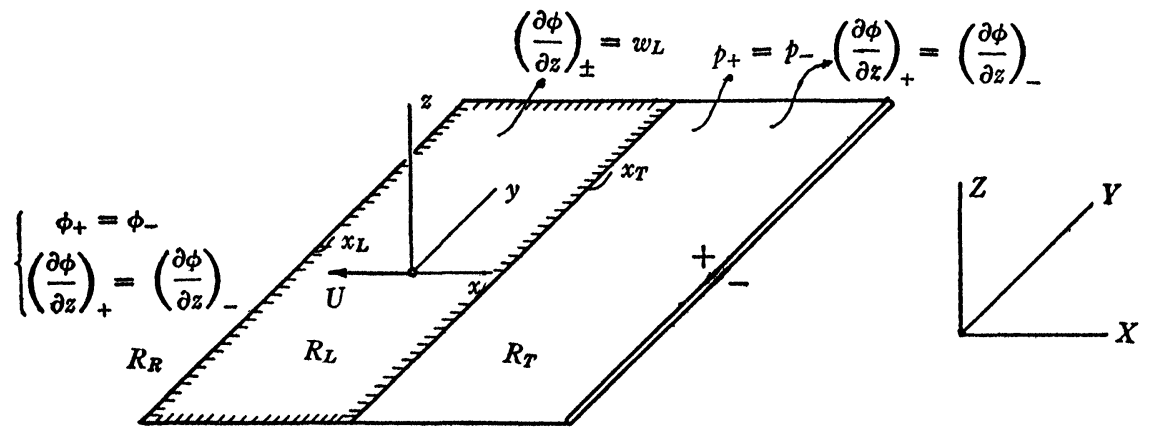

Fig. 2

We shall designate the region occupied by the projection of the lifting surface by $R_{L}$ and the region occupied by the trailing surface of discontinuity by $R_{T}$ (Fig. 2).

From (20) follows that when $a_{0}=\infty$ the differential equation is, for steady as well as for nonsteady motion,

$$
\nabla^{2} \phi=0 .
$$

From (19) follows, for the pressure induced by the motion of the lifting surface,

$$
\frac{p_{i}}{\rho_{0}}=-\left(\frac{\partial \phi}{\partial t}+U \frac{\partial \phi}{\partial x}\right)
$$

If the instantaneous distance of a point of the lifting surface from the $x, y$-plane is designated by $Z(x, y, t)$ we have $F_{L}=F_{L}+f_{L}$ $=z-Z(x, y, t)=0$. Consequently the boundary condition (21) becomes

$$
x, y \text { inside } R_{L}: \frac{\partial \phi}{\partial z}=\frac{\partial Z}{\partial t}+U \frac{\partial Z}{\partial x} \equiv w_{L} .
$$

The transition conditions (22) become 


$$
x, y \text { inside } R_{T}\left\{\begin{array}{l}
\left(\frac{\partial \phi}{\partial z}\right)_{+}=\left(\frac{\partial \phi}{\partial z}\right)_{-} \\
\left(\frac{\partial \phi}{\partial t}+U \frac{\partial \phi}{\partial x}\right)_{+}=\left(\frac{\partial \phi}{\partial t}+U \frac{\partial \phi}{\partial x}\right)_{-}
\end{array}\right.
$$

To equations (27) and (28) is to be added the condition of finite trailing edge velocities

$$
z=0, x=x_{T}(y) ; \quad \nabla \phi \text { finite }
$$

and conditions at infinity which for incompressible flow may be taken in the form

$$
\begin{aligned}
& x=-\infty \\
& z= \pm \infty
\end{aligned}\left\{\nabla \phi=0, \quad \frac{\partial \phi}{\partial t}+U \frac{\partial \phi}{\partial x}=0 .\right.
$$

The above problem is to be understood as a boundary value problem for the exterior of an infinitely thin semi-infinite tube surrounding the regions $R_{L}$ and $R_{T}$, in the sense that (27) holds for $z=+0$ and for $z=-0$. It may be recalled that the main object is the determination of $p_{i+}$ and $p_{i-}$ in $R_{L}$, with $p_{i-}-p_{i+}$ being the lift intensity produced by the motion of the impenetrable surface $F_{L}$.

The form of the boundary conditions (27) to (30) is such that the problem for the exterior of the semi-infinite tube may be transformed by a symmetry consideration into a mixed boundary-value problem for one of the half spaces $z>0$ or $z<0$. This is done by observing that equations (27) and (30) are compatible with the assumption that $\phi$ is an odd function of $z$. If we define a region $R_{R}$ as the $x, y$-plane minus the regions $R_{L}$ and $R_{T}$ and take into account that $\phi$ is continuous except across $R_{L}$ and $R_{T}$ we may replace the boundary conditions (27) and (28) by the following system of conditions at $z=0$ :

$$
\begin{cases}x, y \text { inside } R_{L}: & \frac{\partial \phi}{\partial z}=w_{L} \\ x, y \text { inside } R_{T}: & \frac{\partial \phi}{\partial t}+U \frac{\partial \phi}{\partial x}=0 \\ x, y \text { inside } R_{R}: & \phi=0 .\end{cases}
$$

We then must determine $\phi$ in one of the half-spaces, say $z>0$, with the conditions (29) and (31) at the boundary $z=0$ and with equations (30) giving the conditions at infinity.

Let us remark that explicit solutions of the problem thus formulated are possible in the two-dimensional case by the use of elliptic 
cylinder coordinates, while for the circular plan form wing the use of spheroidal coordinates is appropriate [41, 62]. The problem would be of a standard nature if the conditions in $R_{T}$ were the same as in $R_{R}$. The main difficulty of obtaining an explicit solution comes from the particular form of the boundary condition holding in $R_{T}$ inasmuch as all that can be said on the basis of (31) about the values of $\phi$ in $R_{T}$ is

$$
\phi(x, y, 0, t)=\Phi\left(x-\int U d t, y\right)
$$

where $\Phi$ is an arbitrary function of its two arguments. Compensating for this arbitrariness is, as will be seen, the finiteness condition (29).

6. Integral equation formulation of the problem. As one is interested primarily in the values of $p_{i}$ in $R_{L}$ it suggests itself to derive an integral equation for this quantity. This procedure, adopted by Birnbaum [3], Possio [53], Kuessner [42], and others at the suggestion of Prandtl, and known under the name acceleration potential method, has the advantage that it can be developed without explicit introduction of the trailing surface of discontinuity. It does however have the disadvantage of leading to an integral equation with a distinctly more complicated kernel than the corresponding integral equation for the values of $\partial \phi / \partial x$ in $R_{L}$ which we propose to discuss here. The main advantage of the latter formulation is that it permits immediate recognition of the explicit solvability of the problem of non-steady motion in terms of the solution of the corresponding problem of steady motion.

Setting as an abbreviation

$$
\gamma(x, y, t)=\partial \phi(x, y, 0, t) / \partial x
$$

we have the following representation for $\partial \phi / \partial x$ in terms of the boundary values $\gamma$,

$$
\frac{\partial \phi}{\partial x}=\frac{-1}{2 \pi} \iint \gamma(\xi, \eta, t) \frac{\partial}{\partial z}\left(\frac{1}{r}\right) d \xi d \eta
$$

where

$$
r^{2}=(x-\xi)^{2}+(y-\eta)^{2}+z^{2}
$$

Equation (34) may be converted into an expression for $\partial \phi / \partial z$, by appropriate differentiation and integration, of the following form

$$
\frac{\partial \phi}{\partial z}=\frac{-1}{2 \pi} \iint \gamma(\xi, \eta, t)\left[\int_{-\infty}^{x} \frac{\partial^{2}}{\partial z^{2}}\left(\frac{1}{r^{\prime}}\right) d x^{\prime}\right] d \xi d \eta
$$




$$
\begin{aligned}
=\frac{-1}{2 \pi} \iint \gamma(\xi, \eta, t)\left\{\frac{x-\xi}{r^{3}}\right. \\
\left.+\frac{\partial}{\partial y}\left[\frac{y-\eta}{(y-\eta)^{2}+z^{2}}\left(\frac{x-\xi}{r}+1\right)\right]\right\} d \xi d \eta .
\end{aligned}
$$

Note that when $\partial \gamma / \partial \eta=0$ which corresponds to the assumption of two-dimensional flow the $\eta$-integration in (36) can be carried out with the result that

$$
\frac{\partial \phi(x, z, t)}{\partial z}=-\frac{1}{\pi} \int \frac{\gamma(\xi, t)(x-\xi)}{(x-\xi)^{2}+z^{2}} d \xi
$$

which equation can of course be obtained directly in a simpler manner.

To separate two-dimensional from three-dimensional effects the following transformation is useful. We write (omitting for brevity the $t$ in $\gamma$ )

$$
\gamma(\xi, \eta)=\gamma(\xi, y)+[\gamma(\xi, \eta)-\gamma(\xi, y)] .
$$

Appropriate integration by parts then gives the following relation

$$
\begin{aligned}
\frac{\partial \phi}{\partial z}= & -\frac{1}{\pi} \int \frac{\gamma(\xi, y)(x-\xi)}{(x-\xi)^{2}+z^{2}} d \xi \\
& -\frac{1}{2 \pi} \iint \frac{\partial \gamma}{\partial \eta}\left\{\frac{y-\eta}{(y-\eta)^{2}+z^{2}}\left(\frac{x-\xi}{r}+1\right)\right. \\
& \left.+\frac{(x-\xi)(y-\eta)}{(x-\xi)^{2}+z^{2}}\left(\frac{1}{r}-\frac{1}{|y-\eta|}\right)\right\} d \xi d \eta
\end{aligned}
$$

We must now in equation (38) let $z$ tend to zero and substitute the boundary conditions (31). It is advantageous that (38) is in such a form that, as can be proved, the two limiting processes of integration and of letting $z$ tend to zero can be interchanged, provided the integrals are defined where appropriate as Cauchy principal values. Thus from (31) and (38)

$$
\begin{aligned}
w_{L}(x, y, t)= & -\frac{1}{\pi} \int_{x_{L}(y)}^{x_{T}(y)} \frac{\gamma(\xi, y, t)}{x-\xi} d \xi-\frac{1}{\pi} \int_{x_{T}(y)}^{\infty} \frac{\gamma_{T}(\xi, y, t)}{x-\xi} d \xi \\
& -\frac{1}{2 \pi} \iint_{R_{L}} \frac{\partial \gamma}{\partial \eta}\left\{\frac{1}{y-\eta}+K(x-\xi, y-\eta)\right\} d \xi d \eta \\
& -\frac{1}{2 \pi} \iint_{R_{T}} \frac{\partial \gamma_{T}}{\partial \eta}\left\{\frac{1}{y-\eta}+K(x-\xi, y-\eta)\right\} d \xi d \eta
\end{aligned}
$$


In (39) $x_{L}$ and $x_{T}$ indicate the coordinates of the leading and trailing edge, $\gamma_{T}$ is still to be determined as far as possible from the boundary conditions and $K$ is of the following form

$$
K=\frac{\left((x-\xi)^{2}+(y-\eta)^{2}\right)^{1 / 2}-|y-\eta|}{(y-\eta)(x-\xi)} .
$$

We shall from now on assume for simplicity's sake that the region $R_{L}$ is the rectangle $|x| \leqq b,|y| \leqq s b$ and that the velocity $U$ which occurs in (31) is constant. We further introduce dimensionless variables,

$$
x^{\prime}=x / b, \quad y^{\prime}=y / b, \quad t^{\prime}=\omega t
$$

and a dimensionless parameter $k$ of the form

$$
k=\frac{\omega b}{U} \text {. }
$$

For the case of simple harmonic motion $k$ is referred to as the "reduced frequency" of the motion. We may again for simplicity's sake omit in what follows the primes designating the dimensionless variables.

We then have from (32) that

$$
\gamma_{T}(\xi, y, t)=\frac{\partial \Phi(\xi-t / k, y)}{\partial(\xi-t / k)} .
$$

Furthermore on account of the finiteness condition (29)

$$
\Phi(1-t / k, y)=\int_{-1}^{1} \gamma(x, y, t) d x \equiv \Gamma(t, y) .
$$

Then

$$
\Phi(\xi-t / k, y)=\Gamma(t-k(\xi-1), y)
$$

and with

$$
t-k(\xi-1)=\tau, \quad d(k \xi-t)=-d \tau
$$

we have from (43)

$$
\gamma_{T}(\xi, y, t)=-k \frac{\partial \Gamma(\tau, y)}{\partial \tau} .
$$

We introduce (46) and (47) into (39) and obtain the following form of the integral equation of the problem 


$$
\begin{aligned}
w_{L}(x, y, t)= & -\frac{1}{\pi} \int_{-1}^{1} \frac{\gamma(\xi, y, t)}{x-\xi} d \xi \\
& +\frac{1}{\pi} \int_{-\infty}^{t} \frac{\partial \Gamma}{\partial \tau} \frac{d \tau}{(x-1)-(t-\tau) / k} \\
& -\frac{1}{2 \pi} \int_{-s}^{8} \int_{-1}^{1} \frac{\partial \gamma}{\partial \eta}\left\{\frac{1}{y-\eta}+K(x-\xi, y-\eta)\right\} d \xi d \eta \\
& +\frac{1}{2 \pi} \int_{-\infty}^{8} \int_{-\infty}^{t} \frac{\partial^{2} \Gamma}{\partial \eta \partial \tau}\left\{\frac{1}{y-\eta}\right. \\
& \left.+K\left(x-1-\frac{t-\tau}{k}, y-\eta\right)\right\} d \xi d \tau .
\end{aligned}
$$

For uniform motion we have $\partial \Gamma / \partial \tau=0$ and the second and fourth integrals in (48) are absent. The form of equation (48) indicates clearly the manner in which for non-uniform motion the values of the solution $\gamma(x, y, t)$ depend on the past history of the motion through the cumulative effect of successive changes of

$$
\Gamma(\tau, y)=\int_{-1}^{1} \gamma(x, y, \tau) d x
$$

The quantity $\Gamma$ is one-half of what is usually referred to as the circulation intensity at a station $y=$ const. of the lifting surface.

Equation (48) is now to be solved for $\gamma$, in terms of $w_{L}$ and $\Gamma$. Once this is done $\Gamma$ is found by integration in terms of $w_{L}$ and therewith $\gamma$ is expressed in terms of $w_{L}$ only. The pressure $p_{i}$ at the lifting surface is then, according to (26), of the form

$$
\frac{p_{i}}{\rho_{0} U}=-\left[k \frac{\partial}{\partial t}\left(\int_{-1}^{x} \gamma(\xi, y, t) d \xi\right)+\gamma(x, y, t)\right] \text {. }
$$

The advantage of (48) compared with the corresponding equation for the values of $p_{i}$ lies in the form of the kernel $1 /(x-\xi)$ in the first term. This permits solution of (48) in a manner analogous to what is done for the problem of uniform motion.

7. Solution of the two-dimensional problem. In what follows we wish to describe briefly one of the possible methods of solution of this problem, namely that by L. Schwarz [67]. We shall subsequently indicate how to utilize this method for an approximate solution of the three-dimensional problem.

Introducing the (unessential) restriction of simple harmonic motion we set 


$$
w_{L}=\bar{w}_{L} e^{i t}, \quad \gamma=\bar{\gamma} e^{i t}, \quad \Gamma=\bar{\Gamma} e^{i t}
$$

where the barred quantities are functions of the space coordinates at most. Equation (48) can then be written in the following form

$$
\bar{w}_{L}(x)=-\frac{1}{\pi} \int_{-1}^{1} \frac{\bar{\gamma}(\xi) d \xi}{x-\xi}+\frac{i k}{\pi} \bar{\Gamma} \int_{1}^{\infty} \frac{e^{i k(1-\xi)}}{x-\xi} d \xi .
$$

Equation (52) is solved by means of a pair of inversion formulas of the form

$$
\begin{aligned}
& g(x)=-\frac{1}{\pi} \int_{-1}^{1} \frac{f(\xi)}{x-\xi} d \xi, f(1) \text { finite, } \\
& f(x)=\frac{1}{\pi}\left(\frac{1-x}{1+x}\right)^{1 / 2} \int_{-1}^{1}\left(\frac{1+\xi}{1-\xi}\right)^{1 / 2} \frac{g(\xi)}{x-\xi} d \xi
\end{aligned}
$$

which may be considered as a result of two-dimensional potential theory, as discussed most fully by H. Söhngen. ${ }^{3}$

Application of (53) to (52) leads to the following expression for $\bar{\gamma}$

$$
\begin{aligned}
\bar{\gamma}(x)= & \frac{1}{\pi}\left(\frac{1-x}{1+x}\right)^{1 / 2}\left\{\int_{-1}^{1}\left(\frac{1+\xi}{1-\xi}\right)^{1 / 2} \frac{\bar{\omega}_{L}(\xi)}{x-\xi} d \xi\right. \\
& \left.+i k \Gamma \int_{1}^{\infty}\left(\frac{\xi+1}{\xi-1}\right)^{1 / 2} \frac{e^{i k(1-\xi)}}{x-\xi} d \xi\right\} .
\end{aligned}
$$

The main difficulty from here on is the calculation of the pressure $\bar{p}_{i}$ which according to $(50)$ is given by

$$
\frac{p_{i}}{\rho_{0} U}=-\left[i k \int_{-1}^{x} \bar{\gamma}(\xi) d \xi+\bar{\gamma}(x)\right] \text {. }
$$

Before listing the result of this lengthy and somewhat devious calculation we may indicate the nature of the equation for $\bar{\Gamma}$ which occurs in (54). If we integrate both sides of (54) as follows:

$$
\begin{aligned}
\int_{-1}^{1} \bar{\gamma} d x & =\frac{1}{\pi} \int_{-1}^{1}\left(\frac{1+\xi}{1-\xi}\right)^{1 / 2}\left[\int_{-1}^{1}\left(\frac{1-x}{1+x}\right)^{1 / 2} \frac{d x}{x-\xi}\right] \bar{w}_{L}(\xi) d \xi \\
& +\frac{i k}{\pi} \bar{\Gamma} \int_{1}^{\infty}\left(\frac{\xi+1}{\xi-1}\right)^{1 / 2}\left[\int_{-1}^{1}\left(\frac{1-x}{1+x}\right)^{1 / 2} \frac{d x}{x-\xi}\right] e^{i k(1-\xi)} d \xi
\end{aligned}
$$

and take account of the formulas

$$
\int_{-1}^{1}\left(\frac{1-x}{1+x}\right)^{1 / 2} \frac{d x}{x-\xi}=-\pi, \quad|\xi|<1,
$$

3 Math. Zeit. vol. 45 (1939) pp. 245-264. 


$$
\int_{-1}^{1}\left(\frac{1-x}{1+x}\right)^{1 / 2} \frac{d x}{x-\xi}=-\pi\left(1-\left(\frac{\xi-1}{\xi+1}\right)^{1 / 2}\right), \quad 1 \leqq \xi,
$$

then equation (56) can be written one of the forms

$$
\begin{aligned}
& \bar{\Gamma}=-\int_{-1}^{1}\left(\frac{1+\xi}{1-\xi}\right)^{1 / 2} \bar{w}_{L}(\xi) d \xi \\
&-i k e^{i k} \bar{\Gamma} \int_{1}^{\infty}\left(\left(\frac{\xi+1}{\xi-1}\right)^{1 / 2}-1\right) e^{-i k \xi} d \xi \\
& \bar{\Gamma}=-\int_{-1}^{1}\left(\frac{1+\xi}{1-\xi}\right)^{1 / 2} \bar{w}_{L}(\xi) d \xi \\
& 1+i k e^{i k} \int_{1}^{\infty}\left(\left(\frac{\xi+1}{\xi-1}\right)^{1 / 2}-1\right) e^{-i k \xi} d \xi
\end{aligned}
$$

It is at this stage that a combination of Bessel functions makes its appearance in the theory. The integral in the denominator of (59) is expressible in terms of Hankel functions, as follows:

$$
\begin{aligned}
\int_{1}^{\infty}\left(1-\left(\frac{\xi+1}{\xi-1}\right)^{1 / 2}\right) e^{-i k \xi} d \xi & \\
= & \frac{\pi}{2}\left[H_{1}^{(2)}(k)+i H_{0}^{(2)}(k)\right]+\frac{e^{-i k}}{i k} .
\end{aligned}
$$

In view of $(60) \bar{\Gamma}$ can be written in the alternate form

$$
\bar{\Gamma}=\frac{\frac{1}{\pi} \int_{-1}^{1}\left(\frac{1+\xi}{1-\xi}\right)^{1 / 2} \bar{w}_{L}(\xi) d \xi}{2^{-1} i k e^{i k}\left[H_{1}^{(2)}(k)+i H_{0}^{(2)}(k)\right]} .
$$

Our purpose in outlining in some detail the steps leading from (52) to (61) has been to indicate the nature of some of the more simple transformations in the calculation of the pressure distribution on oscillating airfoils. Considerable care is necessary to arrange the analysis in such a manner that advantage is taken of all possible simplifications. In this way there is found the following expression for $\bar{p}_{i}$ of equation (55)

$$
\begin{aligned}
\frac{\bar{p}_{i}}{\rho_{0} U}= & \frac{1}{\pi} \int_{-1}^{1}\left(\left(\frac{1-x}{1+x}\right)^{1 / 2}\left(\frac{1+\xi}{1-\xi}\right)^{1 / 2} \frac{1}{x-\xi}-i k \Lambda\right) \bar{w}_{L}(\xi) d \xi \\
& +\frac{C(k)-1}{\pi}\left(\frac{1-x}{1+x}\right)^{1 / 2} \int_{-1}^{1}\left(\frac{1+\xi}{1-\xi}\right)^{1 / 2} \bar{w}_{L}(\xi) d \xi .
\end{aligned}
$$


In (62) the function $\Lambda$ is given by

$$
\Lambda(x, \xi)=\frac{1}{2} \ln \frac{1-x \xi+\left(1-x^{2}\right)^{1 / 2}\left(1-\xi^{2}\right)^{1 / 2}}{1-x \xi-\left(1-x^{2}\right)^{1 / 2}\left(1-\xi^{2}\right)^{1 / 2}}
$$

and the function $C$, first introduced by Theodorsen [73], is of the form

$$
C(k)=\frac{H_{1}^{(2)}(k)}{H^{(2)}(k)+i H_{0}^{(2)}(k)} .
$$

The results outlined in this section find their main application in the analysis of airplane flutter. ${ }^{4}$ For this purpose explicit expressions have been obtained by Cicala [7], Kuessner [42], Theodorsen [73], and others for lift and moment amplitudes $\bar{L}$ and $\bar{M}_{a}$ defined by

$$
\bar{L}=2 b \int_{-1}^{1} \bar{p}_{i} d x, \quad \bar{M}_{a}=2 b^{2} \int_{-1}^{1}(x-a) p_{i} d x
$$

and for control-surface hinge moments $\bar{M}_{c}$ defined by

$$
\bar{M}_{c}=2 b^{2} \int_{c}^{1}(x-c) p_{i} d x
$$

for various appropriate forms of $\bar{w}_{L}$.

Plots of representative pressure distributions for various values of the reduced frequency $k$ and for some of the more important forms of $w_{L}$ may be found in a recent paper by Postel and Leppert [55].

We may conclude this section with some remarks concerning the solution of the problem for non-oscillatory motion.

It may readily be seen that the results for simple harmonic motion may be used for Laplace transform analysis by replacing wherever it occurs $i k$ by $-q$ whereupon equations (61) and (62) become relations between Laplace transforms. For applications of the Laplace transform method in this field reference may be made to work by I. E. Garrick [17, 18] and W. R. Sears [70].

Another form of the results consists in integro-differential equations for $\Gamma, L$ and $M_{a}$, without any assumption concerning the form of the solution. The nature of these results may be seen from the simplest of them, the equation determining $\Gamma$. Omitting all but the first two of the integrals on the right of (48) we may obtain the following relation

4 Briefly, the problem of flutter is the determination of those flight speeds at which self-sustained oscillations of a component of the airplane become possible due to the aerodynamic forces produced by an oscillatory motion of this component. 


$$
\begin{aligned}
\int_{-1}^{1}\left(\frac{1+x}{1-x}\right)^{1 / 2} w_{L}(x, t) d x & \\
= & -\frac{1}{\pi} \int_{-1}^{1} \gamma(\xi, t)\left\{\int_{-1}^{1}\left(\frac{1+x}{1-x}\right)^{1 / 2} \frac{d x}{x-\xi}\right\} d \xi \\
& \quad+\frac{1}{\pi} \int_{-\infty}^{t} \frac{d \Gamma}{d \tau}\left\{\int_{-1}^{1}\left(\frac{1+x}{1-x}\right)^{1 / 2} \frac{d x}{x-1-(t-\tau) / k}\right\} d \tau .
\end{aligned}
$$

After evaluating the inner integrals on the right of (66) we are left with an equation of the form

$$
\begin{aligned}
\Gamma(t)+\int_{-\infty}^{t} \frac{d \Gamma}{d \tau}\left[\left(\frac{t-\tau+2 k}{t-\tau}\right)^{1 / 2}\right. & -1] d \tau \\
& =-\int_{-1}^{1}\left(\frac{1+x}{1-x}\right)^{1 / 2} w_{L}(x, t) d x .
\end{aligned}
$$

A formal solution of (67) by Fourier series or Laplace transform methods is again readily obtained. A special case of such a solution is given by (61). Practical applications, especially of the Laplace transform solution, are however not a simple matter, the reason for this being the occurrence of Hankel function combinations in the denominator of the transforms to be evaluated, and the possibility of solving (67) directly by machine methods would be of considerable advantage.

8. Remarks on the problem of tunnel wall interference in the twodimensional theory. A further problem of some interest concerning the two-dimensional theory of oscillating airfoils is the effect of tunnel walls on the pressure distribution $\bar{p}_{i}$. For a wing of chord $2 b$ located at the center of a tunnel of height $2 h$ one finds, using the method of images, that (52) is replaced by the following equation [58]

$$
\bar{w}_{L}(x)=-\frac{1}{\pi} \int_{-1}^{1} \frac{\lambda \bar{\gamma}(\xi) d \xi}{\sinh [\lambda(x-\xi)]}+\frac{i k}{\pi} \bar{\Gamma} \int_{1}^{\infty} \frac{\lambda e^{i k(1-\xi)} d \xi}{\sinh [\lambda(x-\xi)]}
$$

where the parameter $\lambda$ is given by

$$
\lambda=\pi b / 2 h .
$$

Equation (69) may be transformed into an equation of the form (61) by means of the following substitutions ${ }^{5}$

5 This transformation has been used in a study of the corresponding problem of steady flow, where the second integral on the right of (68) is absent, by L. Lees and H. S. Tsien, Journal of the Aeronautical Sciences vol. 12 (1945) pp. 173-187, 202. 


$$
\kappa z=\tanh \lambda x, \quad \kappa \xi=\tanh \lambda \xi, \quad \kappa=\tanh \lambda .
$$

The result is of the form

$$
\begin{aligned}
\frac{\bar{w}_{L}^{*}(z)}{\left(1-\kappa^{2} z^{2}\right)^{1 / 2}}= & -\frac{1}{\pi} \int_{-1}^{1} \frac{\bar{\gamma}^{*}(\zeta)}{\left(1-\kappa^{2} \zeta^{2}\right)^{1 / 2}} \frac{d \zeta}{z-\zeta} \\
& +\frac{i k}{\pi} \bar{\Gamma} \int_{1}^{1 / \kappa} \frac{\exp \left\{i k\left[1-\left(\tanh ^{-1} \kappa \zeta\right) / \lambda\right]\right\}}{\left(1-\kappa^{2} \zeta^{2}\right)^{1 / 2}(z-\zeta)} d \zeta .
\end{aligned}
$$

The solution of (71), which involves elliptic integrals, has not yet been given. An approximate solution, valid for sufficiently small values of $\lambda$, has been obtained in the following way [58]. We set in the interval $|\xi| \leqq 1$

$$
\frac{\lambda}{\sinh \lambda(x-\xi)} \approx \frac{1}{x-\xi}-\frac{\lambda^{2}}{6}(x-\xi) .
$$

The limits of integration in the second integral on the right of (68) preclude the direct use of (72). This difficulty is overcome by writing

$$
\begin{aligned}
& \int_{1}^{\infty} \frac{\lambda e^{-i k \xi} d \xi}{\sinh [\lambda(x-\xi)]} \\
& \quad=\int_{1}^{\infty} \frac{e^{i k \xi} d \xi}{x-\xi}+\int_{1}^{\infty} e^{i k \xi}\left[\frac{\lambda}{\sinh [\lambda(x-\xi)]}-\frac{1}{x-\xi}\right] d \xi
\end{aligned}
$$

and by splitting the second integral on the right of (73) into two integrals as follows, $\int_{1}^{\infty}=\int_{x}^{\infty}-\int_{x}^{1}$. The integral $\int_{x}^{\infty}$ leads to the function

$$
f(\mu)=\int_{0}^{\infty} e^{-i \mu x}\left(\frac{1}{x}-\frac{1}{\sinh x}\right) d x
$$

which has been tabulated. In the integral $\int_{x}^{1}$ one may introduce the approximation (72). In this way the following approximate equation, which takes the place of (68), is obtained:

$$
\begin{aligned}
\bar{w}_{L}(x)= & -\frac{1}{\pi} \int_{-1}^{1} \frac{\bar{\gamma}(\xi) d \xi}{x-\xi}+\frac{i k}{\pi} \bar{\Gamma} \int_{1}^{\infty} \frac{e^{i k(1-\xi)}}{x-\xi} d \xi \\
& +\frac{\lambda^{2}}{6 \pi}\left\{\int_{-1}^{1} \bar{\gamma}(\xi)(x-\xi) d \xi+\bar{\Gamma}\left[\frac{1-e^{i k(1-x)}}{i k}+(1-x)\right]\right\} \\
& +\frac{i k}{\pi} \bar{\Gamma} f\left(\frac{k}{\lambda}\right) e^{i k(1-x)}
\end{aligned}
$$

It is of some interest to observe that the effect of the presence of tun- 
nel walls may, for moderate values of $k$, be appreciably larger than the corresponding effect in the steady-state solution for which $k=0$. It was found in one representative example that when $k=0.25$ the wall effect was twice what it was when $k=0$. This increase of the wall effect will occur when at the same time $k$ is (1) large enough for the trailing surface of discontinuity to be of importance and (2) small enough for the characteristic wavelength in the trailing surface of discontinuity to be appreciably greater than the wing chord.

9. Approximate theory for lifting surfaces of finite span. We now propose to discuss a method of approximate solution of the integral equation (48) for three-dimensional flow from the following point of view. Our object is to reduce (48), which contains double integrals, to such a form that a solution of single integral equations only is required. We shall show that this is possible, provided the "aspect ratio" $s$ is sufficiently large, in such a way that what remains to be found is the solution of a problem of the kind encountered in the two-dimensional theory and the solution of a problem of the kind encountered in the determination of the spanwise lift distribution for a wing in uniform motion according to Prandtl's lifting-line theory. In this analysis we shall restrict attention to the case of simple harmonic motion in the sense of (51). Equation (48) then assumes the following form

$$
\begin{aligned}
\bar{w}_{L}(x, y)= & -\frac{1}{\pi} \int_{-1}^{1} \frac{\bar{\gamma}(\xi, y)}{x-\xi} d \xi+\frac{i k}{\pi} \bar{\Gamma}(y) \int_{1}^{\infty} \frac{e^{i k(1-\xi)}}{x-\xi} d \xi \\
& -\frac{1}{2 \pi} \int_{-8}^{s} \int_{-1}^{1} \frac{\partial \bar{\gamma}}{\partial \eta}\left\{\frac{1}{y-\eta}+K(x-\xi, y-\eta)\right\} d \xi d \eta \\
& +\frac{i k}{2 \pi} \int_{-s}^{s} \int_{1}^{\infty} \frac{d \bar{\Gamma}}{d \eta} e^{i k(1-\xi)}\left\{\frac{1}{y-\eta}\right. \\
& +K(x-\xi, y-\eta)\} d \xi d \eta
\end{aligned}
$$

Our first step is to observe that the function $K$ as given by equation (40) behaves, for $|(x-\xi) /(y-\eta)| \ll 1$, like $2^{-1}(x-\xi) /|y-\eta|$ $\cdot(y-\eta)$ and is therewith in this region small compared with the remaining part of the kernel, $1 /(y-\eta)$. If it is now assumed that $1 \ll s$, then $K$ is small compared with $1 /(y-\eta)$ over most of the region of integration and may over this part of the region be disregarded. There remains the immediate neighborhood of the line $\eta=y$ where this order of magnitude relation does not hold. In order to disregard the con- 
tribution due to $K$ in this region it is observed that $K$ is an odd function of $y-\eta$ and that we expect $\partial \bar{\gamma} / \partial \eta$ to be a slowly varying function of $\eta$. In view of this we expect the contribution due to both $K$ and $(1 /(y-\eta)$ to be negligible. We thus assume that

$$
\int_{-s}^{s} \int_{-1}^{1} \frac{\partial \bar{\gamma}}{\partial \eta}\left\{\frac{1}{y-\eta}+K\right\} d \xi d \eta \approx \int_{-\infty}^{s} \int_{-1}^{1} \frac{\partial \bar{\gamma}}{\partial \eta} \frac{d \xi d \eta}{y-\eta}
$$

and assume that the approximation is justified for "sufficiently" large values of $s$.

Obviously the above argument is not very satisfactory, from a mathematical point of view, but no more rigorous argument has yet been given to justify this, from a practical standpoint rather satisfactory, result. Possibly, equation (77) represents the first step in an asymptotic development in powers of $1 / s$ but this has not yet been proved.

The argument leading to (77) cannot directly be applied to the last integral in (76) since in this integral we do not have the fact that $|(x-\xi) /(y-\eta)|$ is small compared to 1 over most of the region of integration. We proceed instead as follows. Write, with $\xi-x=\lambda$,

$$
\begin{aligned}
\int_{1}^{\infty} e^{-i k \xi}\left\{\frac{1}{y-\eta}\right. & +K(x-\xi, y-\eta)\} d \xi=\int_{x}^{\infty}-\int_{x}^{1} \\
= & \int_{0}^{\infty} e^{-i k(\lambda+x)}\left\{\frac{1}{y-\eta}-K(\lambda, y-\eta)\right\} d \lambda \\
& -\int_{x}^{1} e^{-i k \xi}\left\{\frac{1}{y-\eta}+K(x-\xi, y-\eta)\right\} d \xi .
\end{aligned}
$$

In the second integral on the right we can again neglect the contribution due to $K$, and thus we may write

$$
\begin{aligned}
& \int_{1}^{\infty} e^{-i k \xi}\left\{\frac{1}{y-\eta}+K\right\} d \xi \approx \frac{e^{-i k}-e^{-i k x}}{i k(y-\eta)} \\
& +e^{-i k x} \int_{0}^{\infty} e^{-i k \lambda}\left\{\frac{1}{y-\eta}-\frac{\left(\lambda^{2}+(y-\eta)^{2}\right)^{1 / 2}-|y-\eta|}{(y-\eta) \lambda}\right\} d \lambda .
\end{aligned}
$$

In view of (79) we now define a function $F$ by the relation

$$
F(z)=\frac{|z|}{z} \int_{0}^{\infty} e^{-i \lambda}\left\{\frac{1}{|z|}+\frac{1}{\lambda}-\left(\frac{1}{z^{2}}+\frac{1}{\lambda^{2}}\right)^{1 / 2}\right\} d \lambda
$$

and combine (77), (79), and (80) in order to obtain from (76) the following approximate integral equation of the problem: 


$$
\begin{aligned}
\bar{w}_{L}(x, y)= & -\frac{1}{\pi} \int_{-1}^{1} \frac{\bar{\gamma}(\xi, y) d \xi}{x-\xi}+\frac{i k}{\pi} \Gamma(y) \int_{1}^{\infty} \frac{e^{i k(1-\xi)}}{x-\xi} d \xi \\
& -\frac{e^{i k(1-x)}}{2 \pi} \int_{-s}^{8} \frac{d \bar{\Gamma}}{d \eta}\left\{\frac{1}{y-\eta}-i k F[k(y-\eta)]\right\} d \eta .
\end{aligned}
$$

Equation (81) is the result which it was intended to obtain. The solution of (81) proceeds as follows. One first obtains $\bar{\gamma}(x, y)$, just as in the two-dimensional theory except that now $\bar{\gamma}$ also depends on $d \bar{\Gamma} / d \eta$. One then obtains, by integration of $\bar{\gamma}$, an equation for the determination of $\bar{\Gamma}$ which is, as was previously stated, of the type of the equation for the determination of the spanwise lift-distribution for a wing in uniform motion according to the lifting-line theory. Finally, just as in the two-dimensional theory, an expression is obtained for the pressure distribution $p_{i}$.

It is readily shown, for instance by the procedure leading from (66) to (67), that the equation for $\bar{\Gamma}$ is of the following form:

$$
\bar{\Gamma}(y)+\mu(k) \int_{-s}^{s} \frac{d \bar{\Gamma}}{d \eta}\left\{\frac{1}{y-\eta}-i k F[k(y-\eta)]\right\} d \eta=\bar{\Gamma}^{(2)}(y)
$$

where $\bar{\Gamma}^{(2)}$ is the value of $\bar{\Gamma}$ according to the two-dimensional theory as given by (61) and where the function $\mu$ is defined by

$$
\mu(k)=\frac{J_{0}(k)-i J_{1}(k)}{\pi k\left[H_{0}^{(2)}(k)-i H_{1}^{(2)}(k)\right]} .
$$

An equation corresponding to (82) had been obtained by Cicala [9] on the basis of entirely different considerations involving the effect of lifting lines and horseshoe vortices. Equation (82) as it stands is different from Cicala's to the extent of a difference in the expression for $\mu$. It turns out that Cicala's result can be obtained from (81) by omitting the factor $e^{-i k x}$ in front of the last integral in (81). In aerodynamical language this means that "the downwash induced by the spanwise variation of circulation" is assumed uniform across the chord. This is indeed correct, as has long been known, for the case of uniform motion for which $k=0$. Evidently lifting line considerations do not permit us to determine the chordwise variation of downwash referred to above and in this respect the integral equation method as outlined goes further.

Further work along the lines indicated leads to the result that the effect of three-dimensionality of the flow as determined by the foregoing approximate theory may be incorporated into equation (62) for the pressure distribution $p_{i}$ of the two-dimensional theory by merely 
changing the function $C(k)$ into $C(k)+\sigma$. The term $\sigma$ depends on the data of a given problem in the following manner:

$$
\sigma=\left[\frac{\bar{\Gamma}(y)}{\bar{\Gamma}^{(2)}(y)}-1\right]\left[C(k)+\frac{i J_{1}(k)}{J_{0}(k)-i J_{1}(k)}\right] .
$$

The foregoing theory, up to equation (83) and including expressions for lift $\bar{L}$ and moment $\bar{M}_{a}$ as defined by (65a, b), is essentially that of [56]. In this reference there are also included discussions of earlier work of a related nature by R. T. Jones [33], Kuessner [44], von Borbely [5], and Sears [69]. Extension of the theory to surfaces of non-rectangular plan form and modification of $p_{i}$ by means of the function $\sigma$ was first presented in [57]. Prior to this M. W. Hunter had established that the results of [56] permitted incorporation of the effect of finite span into the expressions for $\bar{L}, \bar{M}_{a}$, and $\bar{M}_{c}$ by means of the function $\sigma[26]$.

A considerable simplification of the present developments as compared with those in [56] and [57] is due to the fact that the basic integral equation of the problem is here taken in the form (76), which is a direct consequence of (38), rather than in that form which corresponds to equation (36).

Methods of analysis and numerical examples of application of this theory may be found in [61]. It may further be mentioned that the analogue of equation (81), for subsonic compressible flow, has recently been obtained [59].

A shortcoming of the approximate theory as discussed is the following. One would expect from an exact solution of the problem that the pressure $p_{i}$ tends to zero, and therewith also circulation $\Gamma$, lift $L$, and moments $M$, as the tip sections are approached. This is indeed the case for lifting surfaces with zero tip chord, such as the elliptical surface. For lifting surfaces with finite tip chord, such as the rectangular surface, one finds however that only $\Gamma_{\text {tip }}$ vanishes as it should whereas $L_{\text {tip }}$ and $M_{\text {tip }}$, while smaller than according to the two-dimensional theory, can not in general be made to vanish. The reason for this difficulty is to be found in the form of the approximate equation (81) in which the effect of spanwise variation of $\bar{\gamma}$ appears solely by way of the average $\bar{\Gamma}$ of $\bar{\gamma}$. A more refined approximate theory undoubtedly requires inclusion of the effects of weighted averages of $\bar{\gamma}$ as well, such as $\int_{-1}^{1} x \bar{\gamma} d x$ and $\int_{-1}^{1} x^{2} \bar{\gamma} d x$, in the integral equation of the problem. With such a refined theory one has reason to expect that tip conditions can be satisfied to a greater degree of approximation than by the present theory, in particular, it will be possible to insure that $L_{\text {tip }}$ and $M_{\text {tip }}$ vanish. 
Development of such a refined approximate theory is believed to be one of the worthwhile future tasks in this field. An application of this thought to the problem of uniform motion may be found in a note in Proc. Nat. Acad. Sci. U. S. A. vol. 35 (1949) pp. 208-215.

\section{REFERENCES AND LITERATURE}

1. M. A. Biot, Some simplified methods in airfoil theory, Journal of the Aeronautical Sciences vol. 9 (1942) pp. 185-190.

2. M. A. Biot and C. T. Boehnlein, Aerodynamic theory of the oscillating wing of finite span, GALCIT Report No. 5 to the Army Air Forces (1942).

3. W. Birnbaum, Das ebene Problem des schlagenden Flïgels, Zeitschrift für Angewandte Mathematik und Mechanik vol. 4 (1924) pp. 277-292.

4. S. von Borbely, Mathematischer Beitrag zur Theorie der Flügelschwingungen, Zeitschrift für Angewandte Mathematik und Mechanik vol. 16 (1936) pp. 1-4.

5. - - Über einen Grenzfall der instationären räumlichen Tragflilgelströmung, Zeitschrift für Angewandte Mathematik und Mechanik vol. 18 (1938) pp. 319-342.

6. - Ü̈ber die Luftkräfte die auf einen harmonisch schwingenden zweidimensionalen Flügel bei Überschallgeschwindigkeit wirken, Zeitschrift für Angewandte Mathematik und Mechanik vol. 22 (1942) pp. 190-205.

7. P. Cicala, L'azione aerodinamiche sui profili di ala oscillante in presenza di corrente uniforme, Memorie della Reale Accademia delle Scienze di Torino (2) vol. 68 (1934-1935) pp. 73-98.

8. - Su moto non stazionario di un'ala di allungamento finito, Rendiconti della R. Accademia Nazionale dei Lincei vol. 25 (1937) pp. 97-102.

9. —_ La teoria e l'espirienza nel fenomeno della vibrazioni alari, L'Aerotechnica vol. 18 (1938) pp. 412-433 [Also NACA T.M. No. 887 (1939)].

10. - Zuschriften an den Herausgeber, Zeitschrift für Angewandte Mathematik und Mechanik vol. 19 (1939) p. 384 and vol. 20 (1940) p. 184.

11. - Lo stato attuale delle ricerche sul moto instazionario di una superficie portante, L'Aerotechnica vol. 21 (1941) pp. 671-685, 759-773.

12. F. Dietze, Zur Berechnung der Auftriebskraft am schwingenden Ruder, Luftfahrtforschung vol. 14 (1937) pp. 361-362.

13. - Die Luftkräfte der harmonisch schwingenden in sich verformbaren Platte (Ebenes Problem), Luftfahrtforschung vol. 16 (1939) pp. 84-96.

14. G. Ellenberger, Bestimmung der Luftkräfte auf einen ebenen Tragflitgel mit Querruder, Zeitschrift für Angewandte Mathematik und Mechanik vol. 16 (1936) pp. 199-226.

15. - Luftkräfte bei beliebiger instationärer Bewegung eines Tragfiigels mit Querruder und bei Vorhandensein von Boen, Zeitschrift für Angewandte Mathematik und Mechanik vol. 18 (1938) pp. 173-176.

16. I. E. Garrick, Propulsion of a flapping and oscillating airfoil, NACA T.R. No. 567 (1936).

17. - On some Fourier transforms in the theory of non-stationary flows, Proceedings of the Fifth International Congress on Applied Mechanics, 1938, pp. 590-593.

18. - On some reciprocal relations in the theory of non-stationary flows, NACA T.R. No. 629 (1938).

19. I. E. Garrick and S. I. Rubinov, Flutter and oscillating airforce calculations for an airfoil in two-dimensional supersonic flow, NACA T.N. No. 1158 (1946).

20. - Theoretical study of airforces on an oscillating or steady thin wing in a supersonic main stream, NACA T.N. No. 1383 (1947). 
21. R. Glaser, Über die Berechnung der Koefficienten einer in der instationären Tragfliggeltheorie auftretenden unendlichen Matrix, Zeitschrift für Angewandte Mathematik und Mechanik vol. 23 (1943) pp. 279-289.

22. H. Glauert, The force and moment on an oscillating airfoil, British A.R.C., R. and M. No. 1242 (1929).

23. J. M. Greenberg, Some considerations on an airfoil in an oscillating stream, NACA T.N. No. 1372 (1947).

24. M. D. Haskind, Oscillations of a wing in a subsonic gas flow (in Russian), Prikl. Math. i Mekh. vol. 11 (1947) pp. 120-146. [Translation A9-T-22 Air Materiel Command].

25. H. Hoenl, Über das Schallfeld einer gleichförmig-translatorisch bewegten punktförmigen Schallquelle, Annalen der Physik vol. 43 (1943) pp. 437-464.

26. M. W. Hunter, Jr., Calculation of the aerodynamic span effect in flutter analysis, M.S. Thesis, Massachusetts Institute of Technology (1944).

27. R. Isaacs, Airfoil theory for flows of variable velocity, Journal of the Aeronautical Sciences vol. 12 (1945) pp. 113-117.

28. - Airfoil theory for rotary wing aircraft, Journal of the Aeronautical Sciences vol. 13 (1946) pp. 218-220.

29. K. Jaeckel, Über die Krafte auf beschleunigt bewegte veränderliche Tragflïgelprofile, Ingenieur-Archiv vol. 9 (1938) pp. 371-395.

30. - Über die Bestimmung der Zirkulationsverteilung fïr den zwei-dimensionalen Tragfilgel bei beliebigen periodischen Bewegungen, Luftfahrtforschung vol. 16 (1939) pp. 135-138.

31. - Zur Theorie der tragenden Linie im Instationären, Luftfahrtforschung vol. 19 (1942) pp. 57-63.

32. R. T. Jones, The unsteady lift of a finite wing, NACA T.N. No. 682 (1939).

33. - The unsteady lift of a wing of finite aspect ratio, NACA T.R. No. 681 (1940).

34. W. P. Jones, Aerodynamic forces on wings in simple harmonic motion, British A.R.C., R. and M. No. 2026.

35. - Theoretical airload and derivative coefficients for rectangular wings, British A.R.C., R. and M. No. 2145.

36. - Wind tunnel interference effect on the values of experimentally determined derivative coefficients for oscillating airfoils, British A.R.C., R. and M. No. 1912.

37. W. P. Jones and S. S. Skan, Calculations of derivatives for rectangular wings of finite span by Cicala's method, British A.R.C., R. and M. No. 1920.

38. T. von Kármán and W. R. Sears, Airfoil theory for non-uniform motion, Journal of the Aeronautical Sciences vol. 5 (1938) pp. 379-390.

39. S. N. Karp, S. S. Shu and H. Weil, Aerodynamics of the oscillating airfoil in compressible flow, Air Materiel Command T.R. No. F-TR-1167-ND (1947) [Also describes unpublished work by Biot, von Borbely, Dietze, Eichler, Frazer, Jordan, Kuessner, Schade, Schwarz, and Wieland].

40. E. A. Krasilschickowa, Disturbed motion of air caused by vibration of a wing moving at supersonic speed (in Russian), Prikl. Math. i Mekh. vol. 11 (1947) pp. 147-164.

41. H. Krienes and T. Schade, Theorie der schwingenden kreisförmigen Tragfläche auf potentialtheoretischer Grundlage, Luftfahrtforschung vol. 19 (1942) pp. 282-291.

42. H. G. Kuessner, Zusammenfassender Bericht über den instationären Auftrieb von Tragfiugeln, Luftfahrtforschung vol. 13 (1936) pp. 410-424.

43. - Das zwei-dimensionale Problem der beliebig bewegten Tragflüche unter Beriicksichtigung von Partialbewegungen der Flilssigkeit, Luftfahrtforschung vol. 17 (1940) pp. 355-361. 
44. - Allgemeine Tragfächentheorie, Luftfahrtforschung vol. 17 (1940) pp. 370-378 [Also NACA T.M. No. 979].

45. - Zuschriften an den Herausgeber, Zeitschrift für Angewandte Mathematik und Mechanik vol. 19 (1939) p. 384 and vol. 20 (1940) p. 184.

46. H. G. Kuessner and L. Schwarz, Der schwingende Flitgel mit aerodynamisch ausgeglichenem Ruder, Luftfahrtforschung vol. 17 (1940) pp. 337-354 [Also NACA T.M. No. 991].

47. C. C. Lin, E. Reissner, and H. S. Tsien, On two-dimensional non-steady motion of a slender body in a compressible fluid, Journal of Mathematics and Physics vol. 27 (1948) pp. 220-231.

48. J. W. Miles, The aerodynamic forces on an oscillating airfoil at supersonic speeds, Journal of the Aeronautical Sciences vol. 14 (1947) pp. 351-357.

49. - Harmonic and transient motion of a swept wing in supersonic flow, Journal of the Aeronautical Sciences vol. 15 (1948) pp. 343-346.

50. R. M. Morris, The two-dimensional theory of moving airfoils, IV, Proc. Roy. Soc. London Ser. A vol. 188 (1947) pp. 439-463.

51. I. A. Panichkin, Forces acting on an oscillating profile in a supersonic gas flow (in Russian), Prikl. Math. i Mekh. vol. 11 (1947) pp. 165-170 [Translation A9-T-21 Air Materiel Command].

52. C. Possio, L'azione aerodinamica sul profilo oscillante alle velocitd ultrasonore, Acta Pontificia Accademia delle Scienze vol. 1 (1937) pp. 93-106.

53. - L'azione aerodinamica sul profilo oscillante in un fluido compressible a velocitd iposonora, L'Aerotechnica vol. 18 (1938) pp. 441-458.

54. - L'azione aerodinamica su una superficie portante in moto oscillatoria, Atti dell'Accademia Nazionale Lincei, Rendiconti (VI) vol. 28 (1938) pp. 194200.

55. E. E. Postel and E. L. Leppert, Jr., Theoretical pressure distributions for a thin airfoil in incompressible flow, Journal of Aeronautical Sciences vol. 15 (1948) pp. 486492.

56. E. Reissner, On the general theory of thin airfoils for non-uniform motion, NACA T.N. No. 946 (1944).

57. - Effect of finite span on the airload distributions for oscillating wings I, NACA T.N. No. 1194 (1947).

58. - Wind tunnel corrections for the two-dimensional theory of oscillating airfoils, Cornell Aeronautical Laboratory Report No. SB-218-5-3 (1947).

59. - Oscillating airfoils of finite span in subsonic compressible flow, NACA T.N. to be published (1949).

60. E. Reissner and S. Sherman, Compressibility effects in futter, Curtiss-Wright Research Lab. Report No. SB-240-S-1 (1944).

61. E. Reissner and J. E. Stevens, Effect of finite span on the airload distribution for oscillating wings II, NACA T.N. 1195 (1947).

62. T. Schade, Theorie der schwingenden kreisförmigen Tragfläche auf potentialtheoretischer Grundlage I, Luftfahrtforschung vol. 17 (1940) pp. 387-400.

63. W. Schmeidler, Schwingenflug mit Wirbelablösung, Zeitschrift für Angewandte Mathematik und Mechanik vol. 17 (1937) p. 361.

64. - Vortrieb und Widerstand, Zeitschrift für Angewandte Mathematik und Mechanik vol. 19 (1939) pp. 65-86.

65. — Zur Theorie des Schwingenfluges, Sitzungsberichte der Berliner Mathematischen Gesellschaft vols. 38, 39 pp. 64-71.

66. L. Schwarz, Berechnung der Funktionen $V_{1}(s)$ und $V_{2}(s)$ für grössere Werte von $s$, Luftfahrtforschung vol. 17 (1940) pp. 362-369. 
67. - Berechnung der Druckverteilung einer harmonisch sich verformenden Tragfäche in ebener Stromung, Luftfahrtforschung vol. 17 (1940) pp. 379-386.

68. - Untersuchung einiger mit den Zylinderfunktionen nullter Ordnung verwandter Funktionen, Luftfahrtforschung vol. 20 (1943) pp. 341-372.

69. W. R. Sears, A contribution to airfoil theory for non-uniform motion, Proceedings of the Fifth International Congress on Applied Mechanics, 1938, pp. 483-487.

70. - Operational methods in the theory of airfoils in non-uniform motion, Journal of the Franklin Institute vol. 230 (1940) pp. 95-111.

71. H. Söhngen, Bestimmung der Auftriebsverteilung für beliebige instationäre Bewegungen (Ebenes Problem), Luftfahrtforschung vol. 17 (1940) pp. 401-419.

72. G. Temple and H. A. Jahn, Flutter at supersonic speeds, British A.R.C., R. and M. No. 2140.

73. T. Theodorsen, General theory of aerodynamic instability and the mechanism of flutter, NACA T.R. No. 496 (1935).

74. T. Theodorsen and I. E. Garrick, Non-stationary flow about a wing-aileron tab combination inclucing aerodynamic balance, NACA T.R. No. 732 (1942).

75. R. Timman, Beschouwingen over de luchtkrachten op trillende vliegtuigvleugels, Dissertation, Technische Hogeschool Delft, 1946, 154 pp.

76. O. (Taussky) Todd, On some boundary value problems in the theory of the nonuniform supersonic motion of an aerofoil, British A.R.C., R. and M. No. 2141.

77. - A boundary value problem for a hyperbolic differential equation arising in the theory of the non-uniform supersonic motion of an aerofoil, Courant Anniversary Volume, 1948, pp. 421-435.

78. H. Wagner, Über die Entstehung des dynamischen Auftriebs von Tragflitgeln, Zeitschrift für Angewandte Mathematik und Mechanik vol. 5 (1925) pp. 17-35.

Massachusetts Institute of Technology 